BIHEP-Th/97-004

\title{
Reexamination of the resonance contributions in
}

\author{
$B \rightarrow X_{s} e^{+} e^{-}$ \\ Dongsheng Du ${ }^{1,2}$ Hongying Jin $^{2} \quad$ Yadong Yang ${ }^{1,2,3} *$ \\ ${ }^{1}$ CCAST (World Laboratory), P.O.Box 8730, Beijing 100080, China \\ ${ }^{2}$ Institute of High Energy Physics, Academia Sinica, P.O.Box 918(4), Beijing 100039, China ${ }^{\dagger}$ \\ 3 Physics Department of Henan Normal University, Xingxiang, Henan, 453002, China
}

\begin{abstract}
With help of the recent developments in the heavy quarkonium physics, we reexamine the long distance(LD) effects in $B \rightarrow X_{s} e^{+} e^{-}$dominantly from the charmonium resonances $J / \Psi$ and $\Psi^{\prime}$ through the decay chains $B \rightarrow X_{s} J / \Psi\left(\Psi^{\prime}\right) \rightarrow X_{s} e^{+} e^{-}$. We find that the resonance to nonresonance interference are reduced substantially.
\end{abstract}

*Email: duds@bepc3.ihep.ac.cn, yangyd@bepc3.ihep.ac.cn

${ }^{\dagger}$ Mailing address 
The rare decay $B \rightarrow X_{s} e^{+} e^{-}$has been the subject of many theoretical studies in the framework of the standard model and its extensions such as the two Higgs doublet models and models involving supersymmetry [1 \&]. It is believed that once $B \rightarrow X_{s} e^{+} e^{-}$is observed, it will offer an useful probe of the standard model and of its extensions. To this end the relevant branching ratio, the dilepton invariant mass distribution and other distributions of interest should be calculated with sufficient precision before reliable conclusion be drawn.

At present, the effective Hamiltonian for $B \rightarrow X_{s} e^{+} e^{-}$decays relevant for scale $\mu \approx m_{\mathrm{b}}$ in which the short distance QCD effects taken into account has been calculated to the nextto-leading order [9 11] in the framework of a renormalization group improved perturbation theory, and the Wilson coefficients in the Hamiltonian are presented to have rather small dependence on $\mu$ and $\Lambda_{\overline{\mathrm{MS}}}$.

The actual calculation of $B \rightarrow X_{s} e^{+} e^{-}$involves not only the evaluation of Wilson coefficients of ten local operators but also the calculation of the corresponding matrix elements of these operators relevant for $B \rightarrow X_{s} e^{+} e^{-}$, which has been studied in HQET including the non-perturbative $\mathcal{O}\left(1 / m_{\mathrm{b}}^{2}\right)$ corrections enhanced the rate for $B \rightarrow X_{s} e^{+} e^{-}$by roughly $4 \%$ [12,13]. A realistic phenomenological analysis should also include the long distance contributions which are mainly due to the $J / \psi$ and $\psi^{\prime}$ resonances 13 17. However, there are large uncertainties in modeling estimation of such effects and further study is needed. In the papers [13 [17], the phenomenological parameter $a_{2}$ has to be input in the sub-amplitude $A(b \rightarrow s J / \Psi)$ to compensate the large discrepancies between theoretical predictions and experimental data for $\operatorname{Br}\left(B \rightarrow J / \Psi X_{s}\right)$. However, such inputs would lead to overestimation of the LD contributions as it will be presented in what follows.

In this paper, we re-examine the LD contributions $B \rightarrow J / \Psi X_{s} \rightarrow X_{s} e^{+} e^{-}$stimulated by the recent significant progress in heavy quarkonium physics [18, 19. We will show that the LD contributions can be substantial reduced when the subamplitude $A(b \rightarrow s J / \Psi)$ decomposed into two different parts which do not interfere with each other: color singlet and color-octet parts as in NRQCD [19] 
We will start with the effective Hermitian given as follows [11]

$$
\mathcal{H}_{\text {eff }}=-\frac{4 G_{F}}{\sqrt{2}} V_{t s}^{*} V_{t b} \sum_{i=1}^{10} C_{i} \mathcal{O}_{i}
$$

where the operator basis is chosen to be

$$
\begin{aligned}
Q_{1} & =\left(\bar{s}_{\alpha} c_{\beta}\right)_{V-A}\left(\bar{c}_{\beta} b_{\alpha}\right)_{V-A}, \\
Q_{2} & =(\bar{s} c)_{V-A}(\bar{c} b)_{V-A} \\
Q_{3} & =(\bar{s} b)_{V-A} \sum_{q}(\bar{q} q)_{V-A}, \\
Q_{4} & =\left(\bar{s}_{\alpha} b_{\beta}\right)_{V-A} \sum_{q}\left(\bar{q}_{\beta} q_{\alpha}\right)_{V-A}, \\
Q_{5} & =(\bar{s} b)_{V-A} \sum_{q}(\bar{q} q)_{V+A}, \\
Q_{6} & =\left(\bar{s}_{\alpha} b_{\beta}\right)_{V-A} \sum_{q}\left(\bar{q}_{\beta} q_{\alpha}\right)_{V+A}, \\
Q_{7} & =\frac{e}{8 \pi^{2}} m_{b} \bar{s}_{\alpha} \sigma^{\mu \nu}\left(1+\gamma_{5}\right) b_{\alpha} F_{\mu \nu}, \\
Q_{8} & =\frac{g}{8 \pi^{2}} m_{b} \bar{s}_{\alpha} \sigma^{\mu \nu}\left(1+\gamma_{5}\right) T_{\alpha \beta}^{a} b_{\beta} G_{\mu \nu}^{a} \\
Q_{9} & =(\bar{s} b)_{V-A}(\bar{e} e)_{V}, \\
Q_{10} & =(\bar{s} b)_{V-A}(\bar{e} e)_{A},
\end{aligned}
$$

where $\alpha$ and $\beta$ denote color indices, $L$ and $R$ denote chiral projections $L(R)=1 / 2\left(1 \mp \gamma_{5}\right)$. The Wilson coefficients can be found in [11,21, 26].

Using the effective Hamiltonian, the SM-based short distance (SD) matrix element for the decay $b \rightarrow s e^{+} e^{-}$can be written as

$$
\begin{aligned}
\mathcal{M}\left(b \rightarrow s e^{+} e^{-}\right)=\frac{G_{F} \alpha}{\sqrt{2} \pi} V_{t s}^{*} V_{t b} & {\left[\left(C_{9}^{e f f}-C_{10}\right)\left(\bar{s} \gamma_{\mu} L b\right)\left(\bar{e} \gamma^{\mu} L e\right)\right.} \\
+ & \left(C_{9}^{e f f}+C_{10}\right)\left(\bar{s} \gamma_{\mu} L b\right)\left(\bar{e} \gamma^{\mu} R e\right) \\
& \left.-2 C_{7}^{e f f}\left(\bar{s} i \sigma_{\mu \nu} \frac{q^{\nu}}{q^{2}} m_{b} R b\right)\left(\bar{e} \gamma^{\mu} e\right)\right] .
\end{aligned}
$$

Introducing

$$
\hat{s}=\frac{q^{2}}{m_{b}^{2}}=\frac{\left(p_{e^{+}}+p_{e^{-}}\right)^{2}}{m_{\mathrm{b}}^{2}}, \quad z=\frac{m_{\mathrm{c}}}{m_{\mathrm{b}}}
$$

and taking the spectator approximation, one finds

$$
\frac{d B r\left(B \rightarrow X_{s} e^{+} e^{-}\right)}{d \hat{s}}=\operatorname{Br}(b \rightarrow c e \bar{\nu}) \frac{\alpha^{2}}{4 \pi^{2}}\left|\frac{V_{t s}}{V_{c b}}\right|^{2} \frac{(1-\hat{s})^{2}}{f(z) \kappa(z)}\left[(1+2 \hat{s})\left(\left|\widetilde{C}_{9}^{e f f}\right|^{2}+\left|\widetilde{C}_{10}\right|^{2}\right)+\right.
$$




$$
\left.4\left(1+\frac{2}{\hat{s}}\right)\left|C_{7}^{(0) e f f}\right|^{2}+12 C_{7}^{(0) e f f} \operatorname{Re} \widetilde{C}_{9}^{e f f}\right]
$$

where

$$
\begin{aligned}
\widetilde{C}_{9}^{e f f}= & \widetilde{C}_{9}^{N D R} \tilde{\eta}(\hat{s})+h(z, \hat{s})\left(3 C_{1}^{(0)}+C_{2}^{(0)}+3 C_{3}^{(0)}+C_{4}^{(0)}+3 C_{5}^{(0)}+C_{6}^{(0)}\right) \\
& -\frac{1}{2} h(1, \hat{s})\left(4 C_{3}^{(0)}+4 C_{4}^{(0)}+3 C_{5}^{(0)}+C_{6}^{(0)}\right) \\
& -\frac{1}{2} h(0, \hat{s})\left(C_{3}^{(0)}+3 C_{4}^{(0)}\right)+\frac{2}{9}\left(3 C_{3}^{(0)}+C_{4}^{(0)}+3 C_{5}^{(0)}+C_{6}^{(0)}\right),
\end{aligned}
$$

and

$$
\begin{aligned}
h(z, \hat{s})= & -\frac{8}{9} \ln \frac{m_{b}}{\mu}-\frac{8}{9} \ln z+\frac{8}{27}+\frac{4}{9} x \\
& -\frac{2}{9}(2+x)|1-x|^{1 / 2} \begin{cases}\left(\ln \left|\frac{\sqrt{1-x}+1}{\sqrt{1-x}-1}\right|-i \pi\right), & \text { for } x \equiv \frac{4 z^{2}}{\hat{s}}<1 \\
2 \arctan \frac{1}{\sqrt{x-1}}, & \text { for } x \equiv \frac{4 z^{2}}{\hat{s}}>1,\end{cases} \\
h(0, \hat{s})= & \frac{8}{27}-\frac{8}{9} \ln \frac{m_{\mathrm{b}}}{\mu}-\frac{4}{9} \ln \hat{s}+\frac{4}{9} i \pi . \\
f(z)= & 1-8 z^{2}+8 z^{6}-z^{8}-24 z^{4} \ln z, \\
\kappa(z)= & 1-\frac{2 \alpha_{s}(\mu)}{3 \pi}\left[\left(\pi^{2}-\frac{31}{4}\right)(1-z)^{2}+\frac{3}{2}\right] \\
\tilde{\eta}(\hat{s})= & 1+\frac{\alpha_{s}(\mu)}{\pi} \omega(\hat{s}),
\end{aligned}
$$

with

$$
\begin{aligned}
\omega(\hat{s})= & -\frac{2}{9} \pi^{2}-\frac{4}{3} \operatorname{Li}_{2}(\hat{s})-\frac{2}{3} \ln \hat{s} \ln (1-\hat{s})-\frac{5+4 \hat{s}}{3(1+2 \hat{s})} \ln (1-\hat{s})- \\
& \frac{2 \hat{s}(1+\hat{s})(1-2 \hat{s})}{3(1-\hat{s})^{2}(1+2 \hat{s})} \ln \hat{s}+\frac{5+9 \hat{s}-6 \hat{s}^{2}}{6(1-\hat{s})(1+2 \hat{s})}
\end{aligned}
$$

Where $f(z)$ and $\kappa(z)$ are the phase-space factor and the single gluon QCD correction to the $b \rightarrow c e \bar{\nu}$ decay 27, 28 respectively. $\tilde{\eta}$ represents single gluon corrections to the matrix element of $Q_{9}$ with $m_{\mathrm{s}}=0$ [10, 29].

Now we implement the LD contributions in $B \rightarrow X_{s} e^{+} e^{-}$. The resonance amplitude, which includes a Breit-Wigner form for the intermediate $\Psi(n S)$ state, is given by the well known formula

$$
A\left(B \rightarrow X_{s} V \rightarrow X_{s} e^{+} e^{-}\right)=\frac{G_{F} \alpha}{\sqrt{2} \pi} V_{t s}^{*} V_{t b}\left(\bar{s} \gamma_{\mu} L b\right)\left(\bar{e} \gamma^{\mu} e\right)
$$




$$
\times\left(C_{1}+\frac{C_{2}}{3}\right) \frac{16 \pi^{2}}{3} \frac{f_{V}^{2} / m_{V}^{2}}{m_{V}^{2}-s-i \Gamma m_{V}},
$$

where $f_{V}$ is the decay constant of vector meson $\mathrm{V}\left(\Psi, \Psi^{\prime}\right)$ determined by

$$
\Gamma\left(V \rightarrow e^{+} e^{-}\right)=\frac{16 \pi \alpha^{2}}{27 m_{V}^{3}} f_{V}^{2}
$$

In the numerical calculations in the literature [13] 17], the combination of the Wilson coefficients $C_{1}+C_{2} / 3$ is treated as a phenomenological parameter $a_{2}$ with a value 0.24 on the consideration of the experiment data $\operatorname{Br}\left(B \rightarrow X_{s} J / \Psi\right)=0.8 \pm 0.08 \%$, which is much larger than the QCD prediction $C_{1}+C_{2} / 3=0.12$, because the usual calculation using the Wilson coefficient would produce very low predictions of the branching ratio $\operatorname{Br}\left(B \rightarrow X_{s} J / \Psi\right)$. However, the theory of heavy quarkonium production and decays has recently undergone a number of significant developments [18, 19], where color-octet mechanism and factorization have been developed. It has been shown that the color-octet contributions compete with the color-singlet contributions in the process $B \rightarrow X_{s} J / \Psi\left(\Psi^{\prime}\right)$ [30 and the predictions agree with the experiment data.

Including the color-octet contributions, one can get

$$
\begin{array}{r}
A\left(B \rightarrow X_{s} \Psi \rightarrow X_{s} e^{+} e^{-}\right)=\frac{G_{F} \alpha}{\sqrt{2} \pi} V_{t s}^{*} V_{t b}\left[C^{s i g}\left\langle X_{s}^{1}\left|\bar{s} \gamma_{\mu} L b\right| B\right\rangle\left\langle\Psi\left|\bar{c} \gamma^{\mu} L c\right| X^{1}\right\rangle\right. \\
\left.+C^{o c t}\left\langle X_{s}^{8}\left|\bar{s} \gamma_{\mu} L T^{a} b\right| B\right\rangle\left\langle\Psi\left|\bar{c} \gamma^{\mu} L T^{a} c\right| X^{8}\right\rangle\right] \\
\times \bar{e} \gamma^{\mu} e \frac{16 \pi^{2}}{3} \frac{f_{\Psi} / m_{\Psi}^{2}}{m_{\Psi}^{2} s-i \Gamma m_{\Psi}}+\left(\Psi \rightarrow \Psi^{\prime}\right),
\end{array}
$$

where $C^{\text {sig }}=\left(C_{1}+C_{2} / 3\right)$ and $C^{\text {oct }}=2 C_{2}$. The term proportional to $C^{\text {sig }}$ is the color singlet amplitude and the term proportional to $C^{\text {oct }}$ is the color-octet amplitude. The color-singlet contribution to $B \rightarrow X_{s} l^{+} l^{-}$can be included directly using eq.(13) as a modification to $\widetilde{C}_{9}^{e f f}$ as the usual cases in $[13$ [17]. However, the color-octet contribution can not be treated in such way, because the color- octet amplitude has different color structure from the SD amplitude in eq.(3) and does not interfere with it within NRQCD [18, 19] just as the cases in 30 32, so, the LD contribution is reduced, especially, in the low $q^{2}$ region. Based on such an observation, we have

$$
\frac{d B_{r}\left(b \rightarrow s e^{+} e^{-}\right)}{d \hat{s}}=B_{r}(b \rightarrow c e \bar{\nu}) \frac{\alpha^{2}}{4 \pi^{2}}\left|\frac{V_{t s}}{V_{c b}}\right|^{2} \frac{(1-\hat{s})^{2}}{f(z) \kappa(z)}[(1+2 \hat{s}) \times
$$




$$
\begin{aligned}
& \left(\left|\widetilde{C}_{9}^{\text {eff }}+Y^{\text {res }}(\hat{s})\right|^{2}+\left|\widetilde{C}_{10}\right|^{2}+\left|C_{\text {oct }}(\hat{s})\right|^{2}\right)+ \\
& \left.4\left(1+\frac{2}{\hat{s}}\right)\left|C_{7}^{(0) e f f}\right|^{2}+12 C_{7}^{(0) e f f} \operatorname{Re}\left[\widetilde{C}_{9}^{\text {eff }}+Y^{\text {res }}(\hat{s})\right]\right]
\end{aligned}
$$

with

$$
\begin{aligned}
Y^{r e s}(\hat{s}) & =C^{s i g} \frac{16 \pi^{2}}{3} \frac{f_{\Psi}^{2} / m_{\Psi}^{2}}{m_{\Psi}^{2}-\hat{s} m_{b}^{2}-i \Gamma_{\Psi} m_{\Psi}}+\left(\Psi \rightarrow \Psi^{\prime}\right), \\
\left|C_{\text {oct }}(\hat{s})\right|^{2} & =C_{\text {oct }}^{2}\left(\frac{16 \pi^{2}}{3}\right)^{2} \frac{3}{2} \frac{\left\langle O_{8}^{\Psi}\left({ }^{3} S_{1}\right)\right\rangle}{\left\langle O_{1}^{\Psi}\left({ }^{3} S_{1}\right)\right\rangle} \frac{f_{\Psi}^{4} / m_{\Psi}^{4}}{\left(\hat{s} m_{b}^{2}-m_{\Psi}^{2}\right)^{2}+\Gamma_{\Psi}^{2} m_{\Psi}^{2}}+\left(\Psi \rightarrow \Psi^{\prime}\right)
\end{aligned}
$$

where $O_{1,8}^{\Psi, \Psi^{\prime}}\left({ }^{3} S_{1}\right)$ are defined in [19]. The other colored $\bar{c} c$ pairs produced at short distance with different quantum number ${ }^{1} S_{0}$ and ${ }^{3} P_{J}$ contribute to $J / \Psi, \Psi^{\prime}$ productions with same Wilson coefficients $C_{o c t}$,

$$
\begin{aligned}
\Gamma(b \rightarrow J / \Psi+X) \propto \quad & 3 C_{\text {oct }}^{2}\left(m_{b}^{2}+8 m_{c}^{2}\right)\left\langle O_{8}^{\Psi}\left({ }^{3} S_{1}\right)\right\rangle \\
& +9 C_{\text {oct }}^{2} m_{b}^{2}\left\langle O_{8}^{\Psi}\left({ }^{3} S_{0}\right)\right\rangle \\
& +6 C_{\text {oct }}^{2}\left(m_{b}^{2}+8 m_{c}^{2}\right)\left\langle O_{8}^{\Psi}\left({ }^{3} P_{1}\right)\right\rangle .
\end{aligned}
$$

The color-octet matrices can be calculated by Lattice calculations or fitted out from experiment data. For simplicity, one can parameterize color octet contributions using only one matrix $\left\langle O_{8}^{\Psi}\left({ }^{3} S_{1}\right)\right\rangle$ in eq(18). Cho and Leibovich [33] have performed a fit to CDF data including the color-octet contribution and found

$$
\begin{aligned}
\left\langle O_{8}^{\Psi}\left({ }^{3} S_{1}\right)\right\rangle & =1.2 \times 10^{-2} \mathrm{GeV}^{3} \\
\left\langle O_{8}^{\Psi^{\prime}}\left({ }^{3} S_{1}\right)\right\rangle & =7.3 \times 10^{-3} \mathrm{GeV}^{3} .
\end{aligned}
$$

Furthermore, using these values, it has been shown that theoretical predictions agree with experiment results of $\operatorname{Br}\left(B \rightarrow \Psi X_{s}\right)$ and $\operatorname{Br}\left(B \rightarrow \Psi^{\prime} X_{s}\right)$ respectively [30, 34]. The colorsinglet matrix elements determined from leptonic decays of $\Psi$ and $\Psi^{\prime}$ are listed as

$$
\begin{aligned}
\left\langle O_{1}^{\Psi}\left({ }^{3} S_{1}\right)\right\rangle & =1.32 \mathrm{GeV}^{3} \\
\left\langle O_{1}^{\Psi^{\prime}}\left({ }^{3} S_{1}\right)\right\rangle & =0.53 G e V^{3} .
\end{aligned}
$$

The numerical results are presented in Fig.1. The thin solid line is the short distance contribution, the dotted line is the result including long distance contribution as the results 
in ref [13 [17], the dotted-dash line is our new result. We can see that the LD effects are significantly reduced, especially in the region of theoretical and experimental interesting $\hat{s}<0.31$ (corresponding the dilepton invariant mass $\sqrt{s}<0.9 m_{\Psi}$ ), where short distance physic might be extracted safely. Here, we recall that there are large uncertainties in the color matrix elements. However, the uncertainties would not spoil our conclusion if the coloroctet mechanism is true and provides solutions to the large heavy quarkonium production data.

Note that in extrapolating the dilepton invariant masses away from the resonance region, no extra $q^{2}$ dependence is included in the $V_{i}-\gamma^{*}\left(q^{2}\right)$ junction. (The $q^{2}$ dependence written explicitly in eq.(3) is due to the Breit-Wigner shape of the resonance). Introducing the momentum dependence junction strength defined by

$$
\left\langle 0\left|\bar{c} \gamma_{\mu} c\right| V(q)\right\rangle=f_{V}\left(q^{2}\right) \epsilon_{\mu}
$$

and using the formula derived by Terasaki [35]

$$
f_{V}\left(q^{2}\right)=g_{V}(0)\left(1+\frac{q^{2}}{C_{V}}\left[d_{V}-h\left(q^{2}\right)\right]\right)
$$

where $C_{\Psi}=0.54, \quad C_{\Psi^{\prime}}=0.77, \quad d_{\Psi}=d_{\Psi^{\prime}}=0.043$ and

$$
h\left(q^{2}\right)=\frac{1}{16 \pi^{2} r}\left[-4-\frac{20 r}{3}+4(1+2 r) \sqrt{\frac{1}{r}-1} \arctan \frac{1}{\sqrt{\frac{1}{r}-1}}\right]
$$

with $r=q^{2} / m_{V}^{2}$ for $0 \leq q^{2} \leq m_{V}^{2}$. For $m_{V}^{2} \leq q^{2}, f_{V}\left(q^{2}\right)=f_{V}\left(m_{V}^{2}\right)$ which is determined by eq(13). Such a consideration leads to another considerable suppression on LD contribution in the low dilepton invariant mass region. The numerical results present in the Fig.1 by a dash line in the region $\hat{s} \leq 0.35$.

In summary, we have investigated the long distance effects in $B \rightarrow X_{s} e^{+} e^{-}$including coloroctet contribution. We have shown that the LD effects are reduced substantially due to the color-octet amplitude not interfering with the SD amplitude and the color-singlet amplitude. This result is important for extracting short distance physics which is a good probe for the physics beyond the Standard Model. Furthermore, if the momentum dependence of the $V_{i}-\gamma^{*}\left(q^{2}\right)$ conversion strength is considered, the long distance effects in the low dilepton 
invariant mass region is negligible small. The main uncertainty in this paper stems from the validity of the application of NRQCD factorization formalism to charmonium production from B meson decays 30 32, 34. NRQCD factorization formalism is well realized in the studies of high $P_{T}$ quarkonium production at hadron colliders, however, in the case of B decays, there is very little excess energy left over. In such a situation, one should pay attention to the validity. To our knowledge, the validity of such a particular application of color-octet heavy quarkonium production ideas has never been justified. Given the validity , another problem still remains obscure. The soft gluon emitted or absorbed by the colored $\bar{c} c$ may obscure dilepton distribution, however, it is unfortunate that little progress has been made in understanding the role played by these soft gluons. Anyway, progress in understanding $B \rightarrow$ Charmonium decays will improve our predictions on $B \rightarrow X_{s} e^{+} e^{-}$.

\section{Acknowledgment}

Y.D. Yang thanks C.S.Kim for helpful discussions and the hospitality of ICTP where this paper is revised. This work is supported in part by the National Natural Science Foundation and the Grant of State Commission of Science and Technology of China.

\section{References}

[1] W. S. Hou, R. I. Willey And A. Soni, Phys. Rev. Lett. 58 (1987) 1608.

[2] B. Grinstein, M. J. Savage and M. B. Wise, Nucl. Phys. B319 (1989) 271.

[3] S. Bertolini, F. Borzumati, A. Masiero and G. Ridolfi, Nucl. Phys. B353 (1991) 591.

[4] A. Ali, T. Mannel, T. Morozumi, Phys. Lett. B273 (1991) 505.

[5] W. Jaus and D. Wyler, Phys. Rev. D41 (1990) 3405.

[6] N. G. Deshpande, K. Panose and J. Trampetić, Phys. Lett. B308 (1993) 322.

[7] A. Ali, G. F. Giudice And T. Mannel, preprint CERN-TH 7346/94, hep-ph/9408213. 
[8] C. Greub, A. IoAnnissian and D. Wyler, preprint ZU-TH 25/94, hep-ph/9408389.

[9] M. Misiak, Nucl. Phys. B393 (1993) 23.

[10] M. Misiak, Nucl. Phys. B461(E) (1995).

[11] A. J. Buras and M. Münz Phys. Rev D52 (1995) 186.

[12] A. Falk, M. Luke and M. J. Savage, Phys. Rev. D49 (1994) 3367.

[13] A. Ali et.al., hep-ph/9609449.

[14] C. S. Lim, T. Morozumi and A. I. Sanda, Phys. Lett. B218 (1989) 343.

[15] N. G. Deshpande, J. Trampetić and K. Panose, Phys. Rev. D39 (1989) 1461.

[16] P. J. O’Donnell and H. K. K. Tung, Phys. Rev. D43 (1991) R2067.

[17] M. R. Ahmady, Phys. Rev. D53 (1996) 2483.

[18] E. Braaten and T.C. Yuan Phys.Rev.Lett 71(1993)1673.

[19] G.T. Bodwin E. Braaten and G.P. Lepage Phys. Rev D51 (1995)1125.

[20] A. J. Buras, M. Jamin and M. E. Lautenbacher, Nucl. Phys. B408 (1993) 209.

[21] A. J. Buras, M. Misiak, M. Münz And S. Pokorski, Nucl. Phys. B424 (1994) 374.

[22] M. Ciuchini, E. Franco, G. Martinelli, L. Reina and L. Silvestrini, Phys. Lett. B316 (1993) 127.

[23] M. Ciuchini, E. Franco, G. Martinelli and L. Reina, Nucl. Phys. B415 (1994) 403.

[24] M. Ciuchini, E. Franco, L. Reina and L. Silvestrini, Nucl. Phys. B421 (1994) 41.

[25] G. Cella, G. Curci, G. Ricciardi and A. Viceré, Nucl. Phys. B431 (1994) 417.

[26] G. Cella, G. Curci, G. Ricciardi and A. Viceré, Phys. Lett. B325 (1994) 227.

[27] N. Cabibbo and L. Maiani, Phys. Lett. B79 (1978) 109.

[28] C. S. Kim and A. D. Martin, Phys. Lett. B225 (1989) 186. 
[29] M. Jeżabek And J. H. Kühn, Nucl. Phys. B320 (1989) 20.

[30] P. Ko, J. Lee and H.S. Song, Phys. Rev D53 (1996)1409.

[31] G.T.Bodwin, E.Brahten and T.C.Yuan, Phys. Rev D46 (1992)3703.

[32] S.Fleming, et al., Phys. Rev D55 (1997)4098.

[33] P. Cho and Leibovich , Phys. Rev D53 (1996)150.

[34] X.He and A.Soni , Phys. Lett B391 (1997)456.

[35] Terasaki, Nuovo Cimento A66(1981)475. 


\section{Figure Captions}

Fig.1. The dileptonic invariant mass spectrum for the decay $B \rightarrow X_{s} e^{+} e^{-}$. The thin solid and the dotted lines correspond to a spectrum without resonances effects, with resonances but phenomenological parameter $a_{2}$ used as in [13 17]. The dotted dash line is the results

including the color-octet effects. The dash line is as same as the dotted-dash line but including the momentum dependent $V-\gamma$ conversion strength effects. 


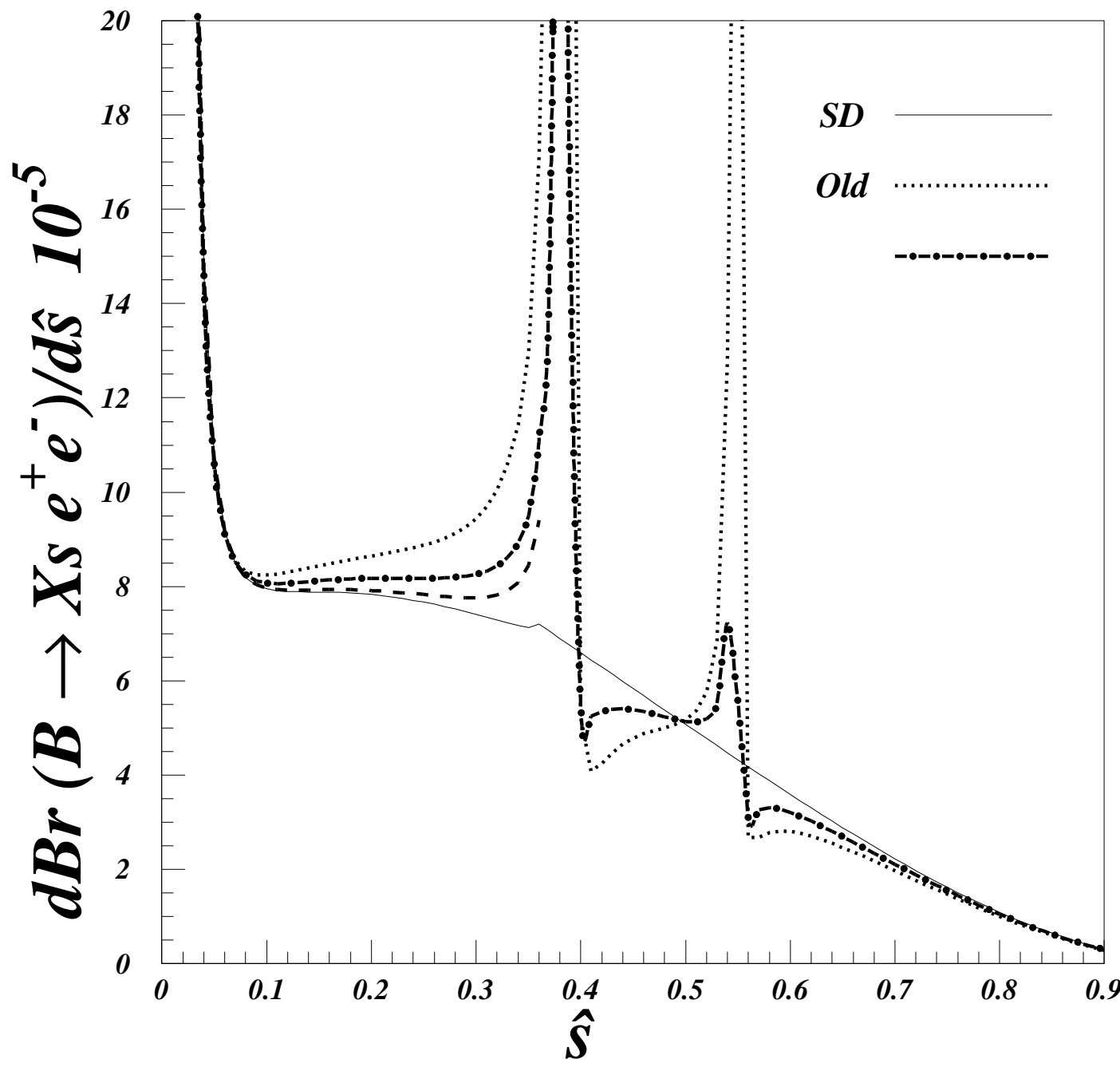

Fig.1 